\title{
Molecular dynamics simulations of two-step process enable room-temperature synthesis of $\alpha-\mathrm{FAPbl}_{3}$
}

\author{
Paramvir Ahlawat ${ }^{\mathrm{a}}$, Haizhou $\mathrm{Lu}^{\mathrm{e}, \mathrm{f}}$, Amita Ummadisingu $^{\mathrm{e}}$, Haiyang Niu ${ }^{\mathrm{b}, \mathrm{d}}$, Michele Invernizzi ${ }^{\mathrm{b}, \mathrm{d}}$, Shaik Mohammed \\ Zakeeruddin $^{\mathrm{e}}$, Anders Hagfeldt ${ }^{\star \mathrm{f}}$, Michael Graetzel ${ }^{\star \mathrm{e}}$, Ursula Rothlisberger ${ }^{\star \mathrm{a}}$, and Michele Parrinello ${ }^{* \mathrm{~b}, \mathrm{~d}}$ \\ ${ }^{\mathrm{a}}$ Laboratory of Computational Chemistry and Biochemistry, Ecole Polytechnique Fédérale de Lausanne, $\mathrm{CH}-1015$ Lausanne, Switzerland; ${ }^{\mathrm{b}}$ Institute of Computational \\ Science, Università della Svizzera Italiana, Ch-6900 Lugano, Switzerland; ${ }^{\mathrm{d}}$ Department of Chemistry and Applied Biosciences, Eidgenossische Technische Hochschule \\ (ETH) Zurich; ' 'Laboratory of Photonics and Interfaces, Ecole polytechnique Fédérale (EPFL) de Lausanne, Lausanne, Switzerland, CH-1015; ${ }^{\mathrm{f} L a b o r a t o r y ~ o f ~}$ \\ Photomolecular Science, Ecole polytechnique Fédérale (EPFL) de Lausanne, Lausanne, Switzerland, $\mathrm{CH}-1015$
}

\begin{abstract}
It is well established that the lack of understanding the crystallization process in two-step sequential deposition has a direct impact on efficiency, stability and reproducibility of perovskite solar cells. Here, we try to understand the solid-solid phase transition occuring during two-step sequential deposition of methylammonium lead iodide and formamidinium lead iodide. Using metadynamics, X-ray diffraction and Raman spectroscopy, we reveal the microscopic details of this process. We find that the formation of perovskite proceeds through intermediate structures and report polymorphs found for methylammonium lead iodide and formamidinium lead iodide. From simulations, we discover a possible crystallization pathway for the highly efficient metastable $\alpha$-phase of formamidinium lead iodide. Guided by these simulations, we perform experiments that results in the room temperature crystallization of $\alpha$-formamidinium lead iodide.
\end{abstract}

Halide Perovskites | Enhanced sampling - Metadynamics | Crystallization | Intermediate phases $\left|\mathrm{MAPbI}_{3}\right| \alpha-\mathrm{FAPbI}_{3} \mid \mathrm{X}_{-}$-ray diffraction | Raman spectroscopy

Perovskite solar cells (PSCs) are one of the most promising and cheap photovoltaic devices(1). However, their widespread application is made difficult by several technological problems related to their long-term stability and processability.

Two-step deposition(2) is one of the main experimental techniques used to fabricate highly efficient and stable PSCs $(3,4)$. In this process, lead iodide $\left(\mathrm{PbI}_{2}\right)$ is deposited first and then converted to perovskite by adding halide salts of monovalent cations such as methylammonium iodide (MAI) and formamidinium iodide (FAI)(5). This process offers several advantages for industrial-scale fabrication(6-8) of larger modules relative to the singlestep spin-coating technology which is limited to smaller devices. However when scaling up, maintaining reproducible high performances and long-term stability is difficult. These problems arise mainly from the lack of control over the fabrication process $(9,10)$. Therefore, it is essential to understand at the atomic-level the mechanism of halide perovskites crystallization.

Among the several perovskites of interest, we study here the two-step fabrication of methylammonium lead iodide $\left(\mathrm{MAPbI}_{3}\right)$ and formamidinium lead iodide $\left(\mathrm{FAPbI}_{3}\right)$. The first one is a well studied system on which many experiments have been performed. The second $\mathrm{FAPbI}_{3}$, is a compound that in its $\alpha-\mathrm{FAPbI}_{3}$ polymorph, a compound that has several attractive features like a $\sim 1.45 \mathrm{eV}$ bandgap, high charge carrier mobility and stability. The practical application of $\alpha-\mathrm{FAPbI}_{3}$ has been hampered by the fact that the $\alpha$-phase is metastable and the thermo- dynamic phase transition requires high temperatures at $\sim 150^{\circ} \mathrm{C}$. We anticipate here that the main result of this paper is the discovery of a low temperature pathway to the fabrication of $\alpha-\mathrm{FAPbI}_{3}$. This has been made possible by a combined experimental and theoretical effort that has uncovered the microscopic details of the crystallization process.

Previous experimental research (11-20) on $\mathrm{MAPbI}_{3}$ has revealed that the two-step process occurs via intercalation of the $\mathrm{MA}^{+}$cations in the $\mathrm{PbI}_{2}$ layers followed by a transformation to the perovskite structure via intermediate phases.

However, these experiments could not resolve the nature of intermediate phases nor elucidate the underlying atomistic mechanism. In order to fill in the details that experiments cannot resolve and obtain an understanding of the microscopic transformation mechanism, we have performed a molecular dynamics investigation. Since the time scale involved in the fabrication process is too large, we have made use of an enhanced sampling technique. In particular, we have used well-tempered metadynamics (WTMetaD)(21, 22). This method allows simulating processes that take place on an extended time scale with affordable computing resources.

We started by an experimental characterization of

"To whom correspondence should be addressed.

ursula.roethlisberger@epfl.ch, anders.hagfeldt@epfl.ch, michael.graetzel@epfl.ch, michele.parrinello@phys.chem.ethz.ch 
$\mathrm{MAPbI}_{3}$ via X-ray and Raman scattering. These experiments provided additional evidence that the picture of the initial intercalation followed by a sequence of intermediates states is correct. We then performed WTMetaD simulations and found that transformation from the intercalated initial structure (see Figure 3a) to the final perovskite arrangement takes place via a sequence of intermediates (see Figure 3b-d). These theoretical results are in line with present and past experiments (23-27).

The highly satisfactory agreement between theory and experiments in the case of $\mathrm{MAPbI}_{3}$ encouraged us to investigate theoretically whether a similar process was operational also for the much less studied $\alpha-\mathrm{FAPbI}_{3}$. This time we started from simulations and indeed discovered that a two-step process is possible at lower temperatures. This was then followed by experiments that confirmed the outcome of the simulations and enabled the low-temperature formation of $\alpha-\mathrm{FAPbI}_{3}$.

\section{Results}

XRD and Raman Spectroscopy. The formation of $\mathrm{MAPbI}_{3}$ in the sequential deposition method was monitored using XRD measurements by examining various samples at different reaction times. See Methods for details about sample preparation.

The XRD spectra of these samples and an unreacted control sample of $\mathrm{PbI}_{2}$ are shown in Supplementary Figure S1a. During the initial seconds of the sequential deposition, we observe a sharpening and narrowing of the (001) peak as the crystallinity of the $\mathrm{PbI}_{2}$ increases, similar to our previous work(28-30). As the reaction progresses, the peak widens becoming less sharp. We further analyzed this process computationally. First we construct models with different size supercells of $\mathrm{PbI}_{2}$ with an increasing number of layers in the (001) direction, depicted in Supplementary Figure S2. We then compute the XRD spectra of these supercells, shown in Supplementary Figure S3 and compare them with the experimental XRD spectra, see Supplementary Figure S1b. We find that during the initial seconds of the dipping, layers of edge-sharing $\mathrm{PbI}_{6}$ octahedra stack over each other in (001) direction of crystalline $\mathrm{PbI}_{2}$. We attribute this fact to intercalation commencing before the formation of $\mathrm{MAPbI}_{3}$. These XRD measurements have provided an interesting first global view of the process.

Next we collected Raman spectra for monitoring the progress of $\mathrm{MAPbI}_{3}$ formation in the sequential deposition reaction. All the Raman spectra are shown in Figure 1. The details are discussed in the Methods section. Here we take a closer look at the peaks at 140 and $143 \mathrm{~cm}^{-1}$, which are associated with the rotational motion of the methylammonium ion in $\mathrm{MAPbI}_{3}(20)$.

The intercalation of the $\mathrm{MA}^{+}$ion into the lattice causes the appearance of the peak at $140 \mathrm{~cm}^{-1}$ at early stages of the reaction (Figure1b, 15s), which then shifts towards $143 \mathrm{~cm}^{-1}$ around $30 \mathrm{~s}$ while growing in intensity. The sig-
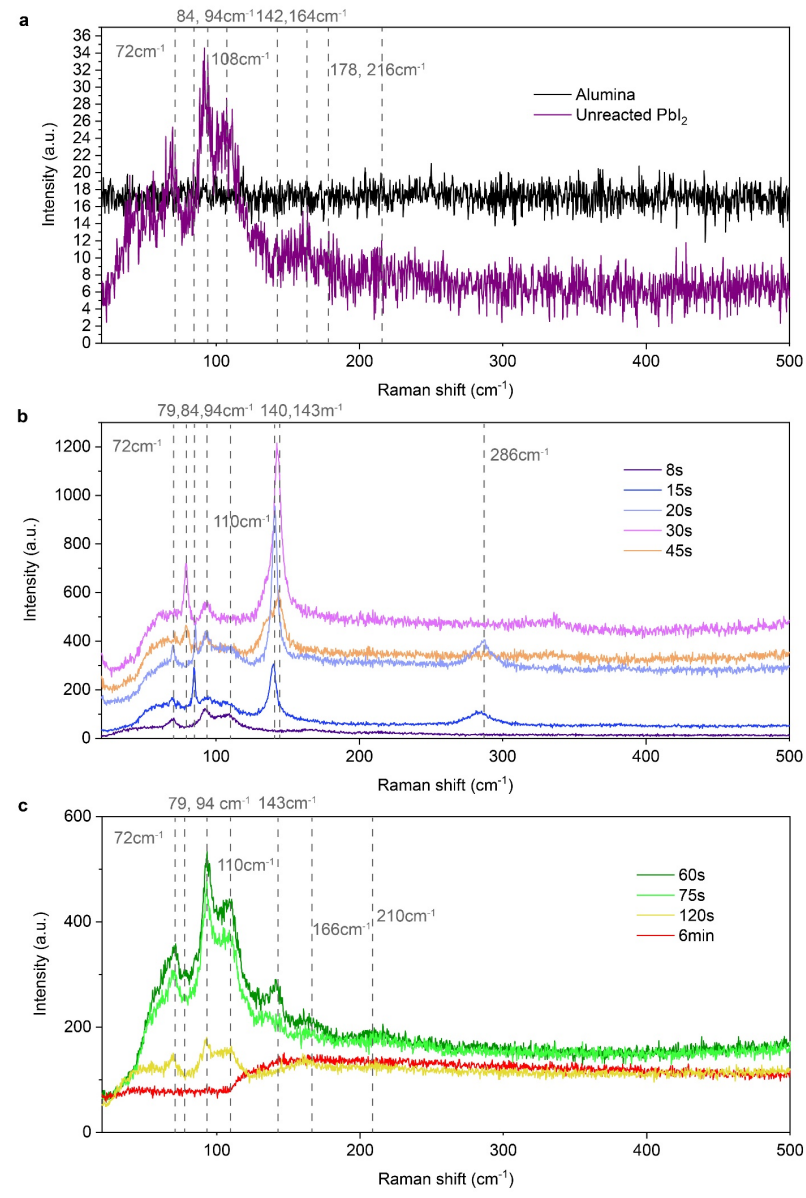

Fig. 1. Raman spectra monitoring the progress of $\mathrm{MAPbl}_{3}$ formation in the sequential deposition reaction. a) Control measurements of the alumina scaffold on glass substrate and the unreacted $\mathrm{Pbl}_{2}$ film. b) samples reacted in $5 \mathrm{mg} / \mathrm{ml} \mathrm{MAI}$ solution after $8,15,20,30$ and $45 \mathrm{~s}$. c) samples reacted in $5 \mathrm{mgml}^{-1} \mathrm{MAl}$ solution after $60,75,120$ s and $6 \mathrm{~min}$.

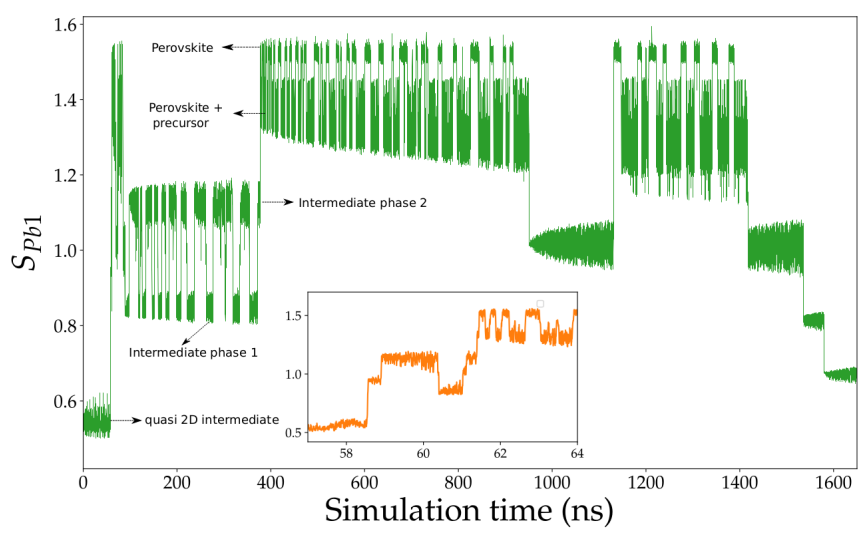

Fig. 2. Structure factor: Evolution of first peak of structure factor of $\mathrm{Pb}^{2+}$ ions $\left(\mathrm{S}_{\mathrm{Pb} 1}\right)$ with WTmetaD simulation at $370 \mathrm{~K}$. Inset is a zoom of $\mathrm{S}_{\mathrm{Pb} 1}$ for the first transition from quasi-2D to $3 \mathrm{D}$. 

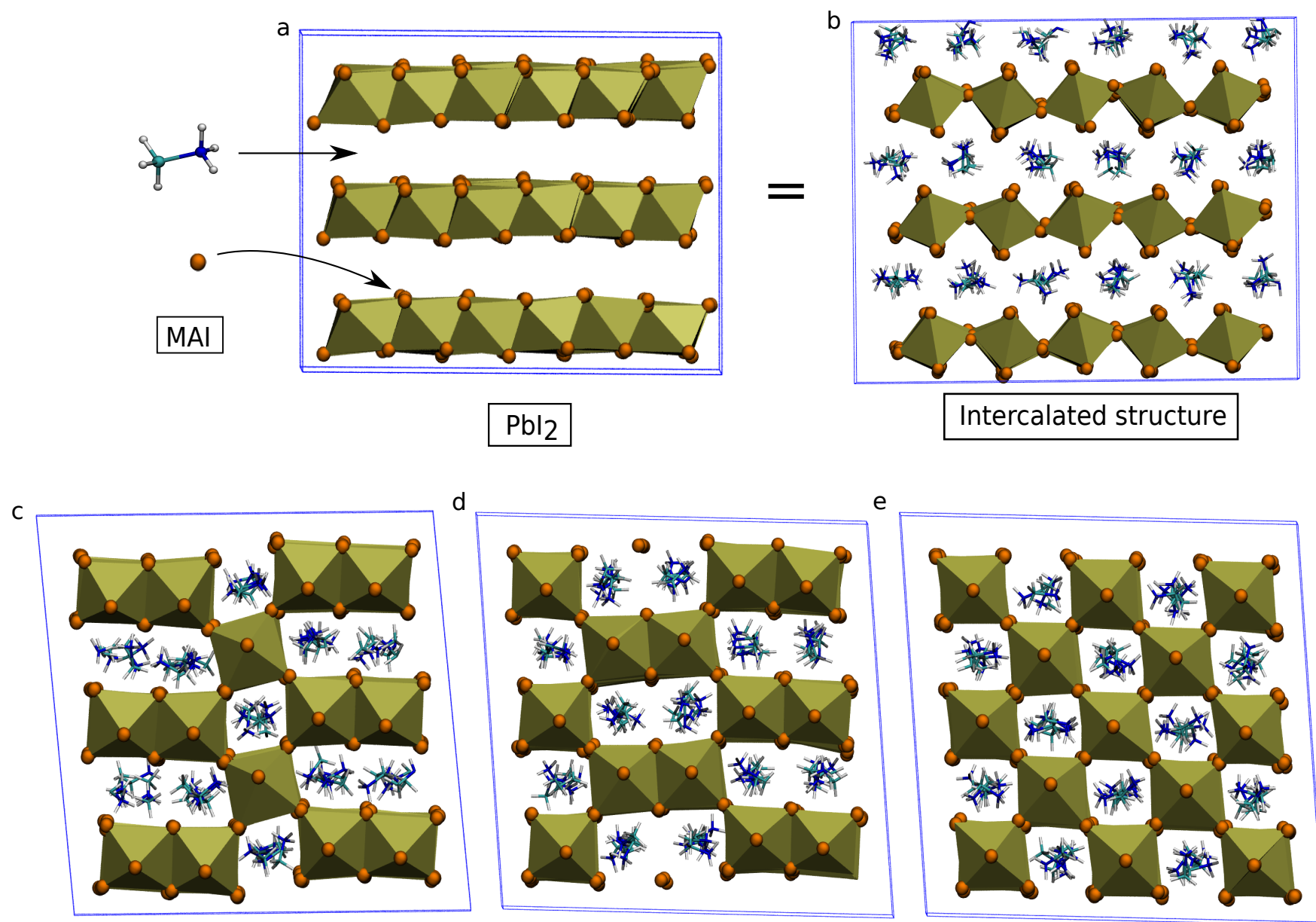

Fig. 3. Phase transition via intermediate phases: Images a and $b$ show the intercalation process. Images $b$ to e display the phase transition from quasi-2D intercalated structure (b) to perovskite phase of $\mathrm{MAPbl}_{3}(\mathrm{e})$. Pb-I complexes are shown as golden polyhedra with $\mathrm{Pb}^{2+}$ in the center and $\mathrm{I}^{-}$as dark orange balls on the corners. $\mathrm{MA}^{+}$ cations are shown with balls and sticks with carbon as light blue, nitrogen as dark blue and hydrogens being white. Similar structures have been observed for other materials such as $\mathrm{MgSiO}_{3}, \mathrm{Fe}_{2} \mathrm{O}_{3}, \mathrm{CslrO}_{3}, \mathrm{AgTaS}_{3}, \mathrm{NaMgF}_{3}, \mathrm{MgGeO}_{3}$. 
nal disappears and broadens at room temperature, as expected for the perovskite structure(31), as the reaction progresses to completion (see data for $75 \mathrm{~s}$ and $6 \mathrm{~min}$ ). These results are highly consistent with the report by Jain et al.(20) who also ascribe these changes to the consumption of $\mathrm{PbI}_{2}$ and the progress of methylammonium iodide intercalation.

Overall, these findings from the experimental investigation suggest the presence of intermediates in the intercalation of the methylammonium ion into the $\mathrm{PbI}_{2}$ and the associated structural reorganization that finally results in the formation of the $\mathrm{MAPbI}_{3}$ perovskite in the sequential deposition reaction.

WTmetaD simulations. From the above mentioned experimental findings, we start from the plausible hypothesis that monovalent cations $\left(\mathrm{MA}^{+}\right)$intercalate between the edge-sharing layers of $\mathrm{Pb}-\mathrm{I}$ octahedra in $\mathrm{PbI}_{2}$ (Fig. 3a) and form a quasi-2D layered structure. We manually construct such a structure by placing $\mathrm{MA}^{+}$ions in between the layers of $\mathrm{PbI}_{2}$ (Figure $3 \mathrm{~b}$ ). The remaining iodide $\left(\mathrm{I}^{-}\right)$from MAI is placed on the Pb-I octahedra to have a final charge-neutral layered $\mathrm{MAPbI}_{3}$ structure. This model is based on experimentally reported intercalated $\mathrm{PbI}_{2}$ structures $(32,33)$. We verify the stability of this structure by carrying out a variable cell enthalpic optimization with classical MD simulations as well as density functional theory (DFT). From both techniques, we find that the structure shown in (Fig. 3b) is stable. Details are presented in the Methods section. Furthermore, we calculate the powder diffraction pattern of the DFT relaxed intercalated structure. We find that the first peak of $\mathrm{PbI}_{2}$ at $\sim 12.7^{\circ}$ shifts to $\sim 11.4^{\circ}$ for the intercalated structure, see Supplementary figure S4. This observation is consistent with the previously reported XRD experiments on two-step deposition(34).

We proceed with the WTmetaD simulations of the transformation of the intercalated $\mathrm{MAPbI}_{3}$ to the perovskite. We prepare two different size supercells of the intercalated structure with 32 and 108 formula units of $\mathrm{MAPbI}_{3}$, respectively. With these supercells, we perform WTMetaD simulations as outlined in the Methods section. These simulations are done at different temperatures ranging from 300 to $420 \mathrm{~K}$ corresponding to typical experimental conditions. For both supercells, we successfully observe the transformation of the starting quasi-2D layered intermediate structure to the 3D corner-sharing perovskite (atomistic views of both simulations are shown in Supplementary Movies M1 and M2). Since a similar transformation mechanism was found in both cases, we only present the results for the larger supercell while the results for the smaller system are given in the SI. Interestingly, we find that the phase transition from quasi-2D to $3 \mathrm{D}$ perovskite proceeds through distinct intermediary structures as shown in Figure $3 \mathrm{~b}$ to e. From these figures and movies: one can see that the intermediate phases are

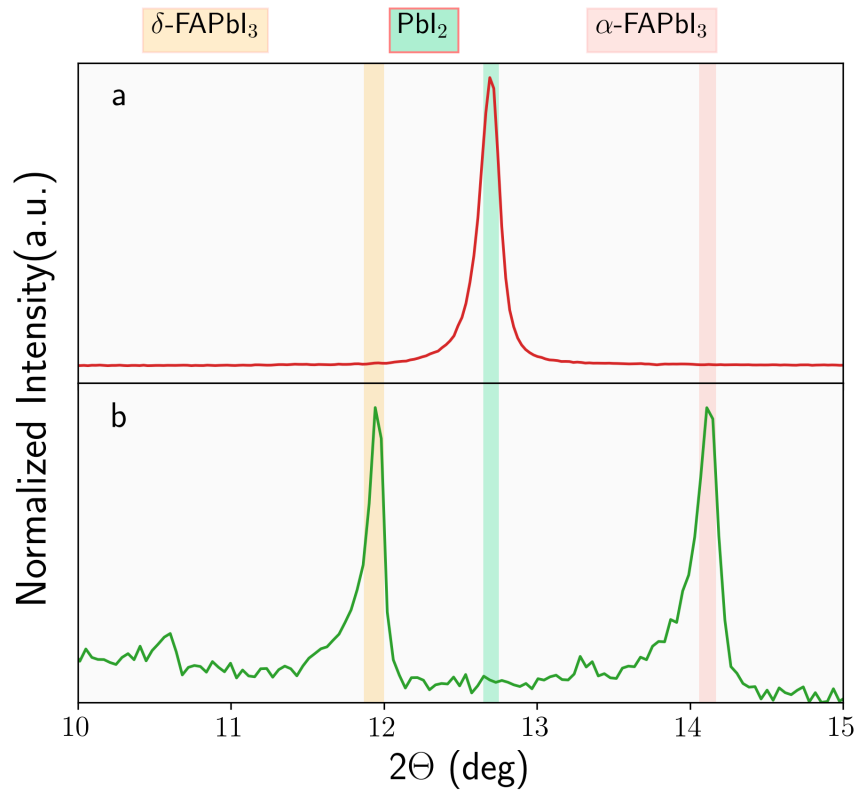

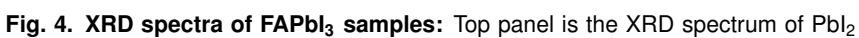
deposited film and bottom panel shows the corresponding data after the FAl loaded onto $\mathrm{Pbl}_{2}$ at room temperature.

formed by rotating $\mathrm{PbI}_{6}$ octahedra over the planes of $\mathrm{PbI}_{2}$ (along the axis of the $\mathrm{Pb}-\mathrm{Pb}$ edge-shared octahedra). This is a general way to the formation of intermediate structures. In the larger supercell more $\mathrm{PbI}_{6}$ octahedra are required to rotate for complete formation of the perovskite structure which leads to formation of more intermediate phases. We quantify this with the evolution of the first peak $\left(\mathrm{q} \approx 10 \mathrm{~nm}^{-1}=2 \Theta \approx 14.1^{\circ}\right)$ of the Debye structure factor $\left(\mathrm{S}_{\mathrm{Pb} 1}\right)$ of $\mathrm{Pb}^{2+}$ ions in cubic perovskites, detailed in the Methods section. Figure 2 shows the evolution of $\mathrm{S}_{\mathrm{Pb} 1}$ for the larger supercell while results for the smaller supercell are shown in Supplementary Figure S5. Moreover, we further explore the free energy landscape and find a variety of different polymorphic structures. We outline all of the observed phases in Supplementary Figure S6. By performing variable cell enthalpic optimization with DFT, we find that all of the structures are stable, see SI. This polymorphic behavior in halide perovskites is related to the existing polytypism in $\mathrm{PbI}_{2}(35,36)$, which also gives rise to these intermediates in crystallization. Many of these polymorphs have a high probability of forming during crystallization of other halide perovskite systems and some of these structures have already been identified experimentally.(23-26) Encouraged by the results for $\mathrm{MAPbI}_{3}$, we proceed to study the phase transition of $\mathrm{FAPbI}_{3}$. Here, the main practical challenge is to find the possible pathways for crystallization of the pure samples of the photoactive $\alpha$ phase. We attempt to solve this problem by first performing simulations and then experiments based on insights from the simulations. We carry out simulations similar to the above reported approach for $\mathrm{MAPbI}_{3}$. First, we construct a quasi-2D $\mathrm{PbI}_{2}$ struc- 
ture intercalated with $\mathrm{FA}^{+}$, similar to the structure in Figure 3b. We perform WTMetaD simulations of two supercells of 32 and 48 formula units of $\mathrm{FAPbI}_{3}$, again , as in the case of $\mathrm{MAPbI}_{3}$, in both cases, we successfully observe the phase transition to the perovskite structure of $\mathrm{FAPbI}_{3}$. An atomic view of this phase transition is shown in Supplementary Movie M3. Remarkably, this simulation reveals a direct path (from the intercalated $\mathrm{PbI}_{2}$ ) to the formation of the metastable phase of $\alpha-\mathrm{FAPbI}_{3}$ without the need for a high-temperature induced transformation via the $\delta$ phase. In particular, our findings suggest that $\alpha-\mathrm{FAPbI}_{3}$ can be formed at lower temperatures from a quasi-2D structure.

We test this newly found route by directly attempting crystallization of $\alpha-\mathrm{FAPbI}_{3}$ at room temperature. At first, we deposit $\mathrm{PbI}_{2}$ layer from a $\mathrm{PbI}_{2}$ solution in N,N-Dimethylformamide (DMF) and Dimethyl sulfoxide (DMSO). After annealing at $70^{\circ} \mathrm{C}$, the $\mathrm{PbI}_{2}$ films are dipped into FAI solution dissolved in isopropanol (IPA) $(20 \mathrm{mg} / \mathrm{ml})$. FAI loading is done at room temperature inside nitrogen filled glove-box. This experiment is shown in Supplementary movie E1. From the movie E1, one can observe that the yellow phase of $\mathrm{PbI}_{2}$ directly transforms into the black phase $\alpha-\mathrm{FAPbI}_{3}$. To gain further insights, we perform XRD measurements on the first $\mathrm{PbI}_{2}$ deposited film and after the loading of FAI. Figure 4 shows that initially $\mathrm{PbI}_{2}$ has formed as indicated by its characteristic peak at $\sim 12.7^{\circ}$. After FAI loading, we see the formation of $\operatorname{cubic}(\alpha)$-phase of $\mathrm{FAPbI}_{3}$ in addition to formation of $\delta$ phase, as indicated by its typical XRD peak $\sim 14.1^{\circ}$. Therefore, it becomes clear that the perovskite structure of $\mathrm{FAPbI}_{3}$ can be formed by FAI intercalation into $\mathrm{PbI}_{2}$ as predicted from our simulations. To scrutinize this further, we also perform another experiment where we load FAI solution onto $\mathrm{PbI}_{2}$ layer during spin-coating process. At difference to the two-step sequential deposition, in this process, we do not observe the formation of the black perovskite phase of $\mathrm{FAPbI}_{3}$. This can be due to the fact that the $\mathrm{PbI}_{2}$-layered structure gets destroyed while spinning the substrate and intercalation of FAI becomes difficult. Further optimization of this process will help in the formation of pure $\alpha-\mathrm{FAPbI}_{3}$ based solar cell modules.

In the end, we point out that the phase transition from a quasi-2D to $3 \mathrm{D}$ perovskite structure via rotation of $\mathrm{PbI}_{6}$ octahedra is a general pathway to form the perovskite structure. This theoretical result can be useful as such a quasi-2D phase can be tailored with additives to synthesize high-quality and stable $\alpha-\mathrm{FAPbI}_{3}$. One example are anion additives such as cloride, which is widely known to modify the crystallization process and has become a commonly used additive to make high efficiency $\alpha-\mathrm{FAPbI}_{3}$ based solar cells. Recent $\mathrm{XRD}(37)$ and cryo-electron microscopy (cryo-EM)(38) experiments have shown that chloride induces a quasi-2D like intermediate phase which can help in nucleation of
$\alpha-\mathrm{FAPbI}_{3}$. Therefore, the current theoretical approach presented here can be applied to unravel the effects of different additives. Moreover, the reverse phase transition from 3D to quasi-2D might also be present during degradation leading to $\mathrm{PbI}_{2}$.

Conclusions. By using WTmetaD, we explore the phase transition paths in the two-step deposition of halide perovskites. Combining simulations and experiments, we reveal the intricate details of the phase transition of $\mathrm{MAPbI}_{3}$ and $\mathrm{FAPbI}_{3}$. We find a low temperature crystallization pathway for the $\alpha-\mathrm{FAPbI}_{3}$. Our combined experimental and simulation study will help in designing better industrial scale processing techniques (39) for $\mathrm{MAPbI}_{3}$ and $\mathrm{FAPbI}_{3}$. In the end, we point out that other Raman studies $(33,40)$ have also demonstrated the $\mathrm{PbI}_{2}$ intercalation with other organic molecules such as ammonia, ethylamine $\left(\mathrm{C}_{2} \mathrm{H}_{5} \mathrm{NH}_{2}\right)$, butylamine $\left(\mathrm{C}_{4} \mathrm{H}_{9} \mathrm{NH}_{2}\right)$, and even larger ones such as cyclohexenyl ethyl ammonium $\left(\mathrm{C}_{6} \mathrm{H}_{9} \mathrm{C}_{2} \mathrm{H}_{4} \mathrm{NH}_{3}{ }^{+}\right)$. Therefore, our combined experimental and simulations study can be extended to study the formation of other systems such as lower-dimensional (2D) perovskites as well.

\section{Materials and Methods}

We use a fixed point charge model for $\mathrm{MAPbI}_{3}$ (41) with $1.0 \mathrm{~nm}$ cutoff for nonbonded interactions. We take the $\mathrm{PbI}_{2}$ parameters from this force field and prepare the force fields for $\mathrm{FAPbI}_{3}$ by mixing rules. In variable cell NPT simulations, this force fields gives stable delta and cubic phases and can also reproduce all other experimentally known $4 \mathrm{H}$ and $6 \mathrm{H}$ polytypes of $\mathrm{FAPbI}_{3}$. Electrostatics are treated with the particle-particleparticle-mesh Ewald method and the SHAKE algorithm(42) is used to constrain covalent bonds to hydrogen. All systems are simulated with the Large-scale Atomic/Molecular Massively Parallel Simulator(LAMMPS) code (31 Mar 2017)(43). We first minimize all systems with a conjugate gradient algorithm keeping a tolerance of maximum force of $10^{-3} \mathrm{kcal} / \mathrm{molA}$. After minimization, we perform a 100ps NVT equilibration run. All production run simulations presented in this work were carried out in the isothermal-isobaric ensemble with a velocity re-scaling thermostat (44) of relaxation time $0.1 \mathrm{ps}$ and a Parrinello-Rahman barostat (45). All WTmetaD simulations are performed with PLUMED 2.4 (46).

DFT calculations. We use Quantum Espresso (47) for geometry optimization with Generalized Gradient Approximation (GGA) of Kohn-Sham DFT, namely the Perdew-Burke-Ernzerhof (PBE) functional revised for solids(PBEsol)(48). For the layered structures, we use the PBE functional(49) with Grimme D2(50) dispersion corrections for van der Waals interactions. In all DFT calculations, we use ultra-soft pseudo-potentials with a plane wave basis set of 60 Ry kinetic energy cutoff and $420 \mathrm{Ry}$ density cutoff. All structure files are reported in the SI.

Collective variables. In this study we use an experimentally measurable quantity, the structure factor (51) as a collective 


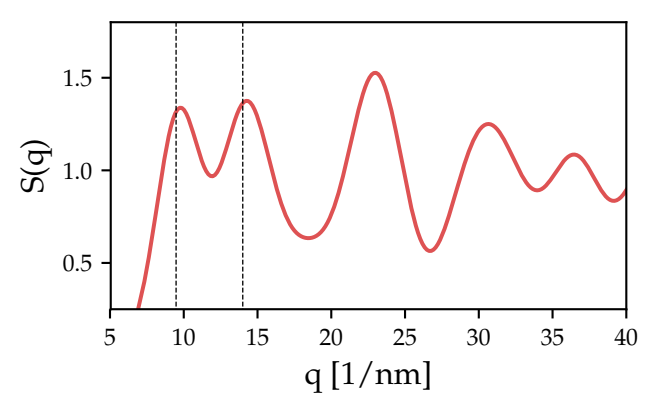

Fig. 5. Debye structure factor of $\mathrm{Pb}^{2+}$ ions in cubic phase of $\mathrm{MAPbl}_{3} . \mathrm{MA}^{+}$have a similar profile due to the symmetry of the cubic phase.

variable $(\mathrm{CV})$. In particular we employ the Debye form (52) of structure factor $(\mathrm{S}(\mathrm{q}))$ :

$$
S(q)=1+\frac{1}{N} \sum_{i=1}^{N} \sum_{j \neq i}^{N} \frac{\sin \left(q r_{\mathrm{ij}}\right)}{q r_{\mathrm{ij}}}
$$

In this equation the sum is done over neighbors of atom i which are contained in a sphere of radius $r_{c}$. As previously done we have added a damping function to remove the termination effects from a finite cutoff. Further details are provided in Refs. (51). However, contrary to previous applications, perovskites are multicomponent systems. In order to deal with this, we use a linear combination of the first two peaks of the structure factors of individual species $\mathrm{Pb}^{2+}$ and $\mathrm{MA}^{+}$, as shown in Figure 5. We design this $\mathrm{CV}$ based on two main factors: (a) $\mathrm{Pb}^{2+}$ are the heaviest atoms and contribute most to XRD spectra and (b) our previous simulations (53) have revealed that the monovalent cations $\left(\mathrm{MA}^{+}\right.$and $\left.\mathrm{FA}^{+}\right)$play an important role in the crystallization process of halide perovskite. Furthermore, these are also the peaks which are assigned to identify perovskite formation in GIWAX experiments. We represent the $\mathrm{MA}^{+}$and $\mathrm{FA}^{+}$cations with the center of $\mathrm{C}-\mathrm{N}$ bond similar to our previous study (53).

$$
C V=S_{\mathrm{Pb} 1}+S_{\mathrm{Pb} 2}+S_{\mathrm{MA} 1}+S_{\mathrm{MA} 2}
$$

We use this $\mathrm{CV}$ to perform the phase transition from the quasi-2D phase to 3D-perovskite. In this study, we have focused on the phase transition of halide perovskites. A similar methodology can also be used to study the phase transitions in many other perovskites systems such as $\mathrm{MgSiO}_{3}(54)$ which is important for earth seismic activities and perovskite solid electrolytes which are promising materials for next-generation $\mathrm{Li}^{+}$ion solid-state batteries (55).

\section{Experimental Methods:.}

Sample preparation: $\mathrm{MAPbI}_{3}$ samples were prepared first by depositing a $\mathrm{PbI}_{2}$ layer directly on glass substrates for the XRD measurements. Samples used for the Raman spectroscopy were made on glass with a mesoporous layer of $23 \mathrm{~nm}$ particles of alumina on them. A $1.3 \mathrm{M} \mathrm{PbI}_{2}$ solution in $\mathrm{DMF}$ was spin coated and the film was then heated at $70{ }^{\circ} \mathrm{C}$ for $10 \mathrm{~min}$. This was followed by the reaction with $5 \mathrm{mg} / \mathrm{ml}$ methylammonium iodide in isopropanol at room temperature, where the sample was dipped in the solution for different periods of time. The final steps were washing in pure isopropanol to halt the reaction and heating at $70{ }^{\circ} \mathrm{C}$ for $10 \mathrm{~min}$.

For $\mathrm{FAPbI}_{3}$ samples, 1.3M $\mathrm{PbI}_{2}$ solution (DMF:DMSO $=9: 1)$ was spin coated at a speed of $2000 \mathrm{rpm} / \mathrm{min}$ for 30 seconds. Then, the $\mathrm{PbI}_{2}$ layer was annealed at 70 degree $\mathrm{C}$ for 1 minute. After that, the $\mathrm{PbI}_{2}$ layer was dipped inside a IPA solution of FAI $(20 \mathrm{mg} / \mathrm{mL})$ for different times.

$\boldsymbol{X R D}$ experiments: $\mathrm{XRD}$ data is measured in the BraggBrentano geometry using a Bruker D8 Advance powder X-ray diffractometer. The setup has $\mathrm{Cu} \mathrm{K} \alpha(\lambda=1.54 \AA)$ radiation and operates in reflection, theta-theta mode (fixed sample) with a $2 \mathrm{D}$ strip detector. A beam mask of $1 \mathrm{~cm}$ was chosen to suitably irradiate the films being measured. A step size of $0.01^{\circ}$ was used for measurement and time/step was $0.15 \mathrm{~s}$.

Raman spectroscopy: Raman spectroscopy was conducted on a HORIBA LabRAM HR Raman spectrometer. A $532 \mathrm{~nm}$ green laser was used for excitation. The focal spot size was about $10 \mu \mathrm{m}$ using a 50x long working distance objective. Previous reports(56) have demonstrated the degradation of the perovskite into $\mathrm{PbI}_{2}$ under high laser intensities. A neutral density filter (OD 3.0) was used to attenuate the laser intensity to avoid damage to the sample.

Raman spectra were collected for monitoring the progress of $\mathrm{MAPbI}_{3}$ formation in the sequential deposition reaction. Figure 1a shows spectra for control samples of the alumina scaffold on the glass substrate and an unreacted $\mathrm{PbI}_{2}$ film. Figure $1 \mathrm{~b}$ shows spectra for samples reacted in $5 \mathrm{mg} / \mathrm{ml}$ MAI solution for 8, 15, 20, 30 and 45s, while Figure 1c shows those for samples reacted for $60,75,120 \mathrm{~s}$ and $6 \mathrm{~min}$.

Figure 1a shows that the control measurement on the substrate shows no peaks in the range of interest so its contribution to the Raman signal in the rest of the measurements can be neglected under similar measurement conditions. The $2 \mathrm{H}$ polytype of $\mathrm{PbI}_{2}$ has only three atoms in each unit cell giving rise to 9 degrees of vibrational motion. The spectrum for the unreacted $\mathrm{PbI}_{2}$ sample shows that peaks at $72 \mathrm{~cm}^{-1}$ (a doubly degenerate $\mathrm{E}_{\mathrm{g}}$ band) and $94 \mathrm{~cm}^{-1}$ (a symmetric stretch $\mathrm{A}_{1 \mathrm{~g}}$ ) due to the shearing motion of two iodine layers are present as expected. Additional peaks expected for $\mathrm{PbI}_{2}$ nanoparticles are detected at 84 and $108 \mathrm{~cm}^{-1}$. Peaks that are double in energy of $72,84,94$ and $108 \mathrm{~cm}^{-1}$ absorptions are also observed at $142,164,178$ and $216 \mathrm{~cm}^{-1}$ and attributed to the overtones of the fundamentals(57).

Now we discuss the Raman spectra of samples reacted with methylammonium iodide. These samples are firstly expected to show indications of the intercalation of the methylammonium ion into the $\mathrm{PbI}_{2}$ lattice as discussed in the XRD results, followed by structural reorganization to form the perovskite. In the Raman spectra for the hybrid perovskite of interest, due to the considerable difference in mass between the organic and inorganic constituents, the spectral features at different energies are associated with different dynamic processes in the perovskite lattice. The features observed at energies below 200 $\mathrm{cm}^{-1}$ are ascribed to a combination of $\mathrm{Pb}-\mathrm{I}$ cage modes and the associated coupled motion of the $\mathrm{MA}^{+}$ions. Those signals above $200 \mathrm{~cm}^{-1}$ are assigned to the molecular vibrations of the cations(5). However, in the data shown in Figure 1c for a sample reacted for $6 \mathrm{~min}$, consisting of fully reacted perovskite in the tetragonal phase, very few features are distinguishable as anticipated at room temperature(31). Nevertheless, the 
data set consisting of the remaining samples shows interesting changes, particularly at energies below $200 \mathrm{~cm}^{-1}$, as the formation of perovskite progresses.

Figure $1 \mathrm{~b}$ shows the intensification of the peak at $79 \mathrm{~cm}^{-1}$ corresponding to the perturbed asymmetric breathing mode of a $\left(\mathrm{MAPbI}_{3}\right)_{4}$ cluster with reaction time(20). It also shows a sharp peak at $84 \mathrm{~cm}^{-1}$ at $30 \mathrm{~s}$ which weakens at $45 \mathrm{~s}$, while the peak at $94 \mathrm{~cm}^{-1}$ strengthens as the reaction progresses beyond this point. From DFT calculations reported in the literature(20), the former can be assigned to the symmetric breathing mode of the $\mathrm{PbI}_{6}$ octahedra and the latter to the perturbed symmetric breathing mode of a $\left(\mathrm{MAPbI}_{3}\right)_{4}$ cluster. These peaks, particularly the $\mathrm{Pb}-\mathrm{I}$ breathing mode at $94 \mathrm{~cm}^{-1}$, vanish as the perovskite formation reaction progresses (Figure $1 \mathrm{c}$, see data for $75 \mathrm{~s}, 120 \mathrm{~s}$ and $6 \mathrm{~min})$.

Acknowledgements. PA is sincerely thankful to Prof. David Mitzi and Prof. Costas Stoumpos for highly insightful discussions. This research is funded by Swiss National Science Foundation through the NCCR MUST and individual grant 200020-185092. M.P. acknowledge funding from European Union Grant No. ERC-2014-AdG$670227 /$ VARMET. The computational time for this work was provided by the SCITAS supercomputers at EPFL and CSCS, the Swiss National Supercomputing Centre.

Author Contributions. PA conceived and conceptualized research. PA designed and perfomed all the simulations. $\mathrm{AU}$ designed and performed experiments on $\mathrm{MAPbI}_{3}$. PA and HL designed and peformed experiments on $\mathrm{FAPbI}_{3}$. PA, HN, MI and MP developed new anaytical tools and analyzed simulations data. UR, SMZ, AH, MG and MP guided research. PA and MP wrote the first draft. All authors discussed the results and wrote the manuscript.

Conflict of Interest. The authors declare no conflict of interest.

Note. AU is currently affiliated with Department of Physics, Cavendish Laboratory, Cambridge, UK. Haiyang Niu is currently affiliated with State Key Laboratory of Solidification Processing, International Center for Materials Discovery, School of Materials Science and Engineering, Northwestern Polytechnical University, Xian 710072, P.R. China.

1. Kojima A, Teshima K, Shirai Y, Miyasaka T (2009) Organometal halide perovskites as visible-light sensitizers for photovoltaic cells. Journal of the American Chemical Society 131(17):6050-6051.

2. Burschka J, et al. (2013) Sequential deposition as a route to high-performance perovskitesensitized solar cells. Nature 499(7458):316-319.

3. Jiang $Q$, et al. (2019) Surface passivation of perovskite film for efficient solar cells. Nature Photonics 13(7):460-466.

4. Yang WS, et al. (2017) lodide management in formamidinium-lead-halide-based perovskite layers for efficient solar cells. Science 356(6345):1376-1379.

5. Liang K, Mitzi DB, Prikas MT (1998) Synthesis and characterization of organic- inorganic perovskite thin films prepared using a versatile two-step dipping technique. Chemistry of materials 10(1):403-411.

6. Razza S, et al. (2015) Perovskite solar cells and large area modules $(100 \mathrm{~cm} 2)$ based on an air flow-assisted pbi2 blade coating deposition process. Journal of Power Sources 277:286291.

7. Li P, et al. (2018) Inkjet manipulated homogeneous large size perovskite grains for efficient and large-area perovskite solar cells. Nano Energy 46:203-211.

8. Park NG, Zhu K (2020) Scalable fabrication and coating methods for perovskite solar cells and solar modules. Nature Reviews Materials pp. 1-18.
9. Minemawari H, et al. (2011) Inkjet printing of single-crystal films. Nature 475(7356):364-367.

10. Bi D, et al. (2016) Polymer-templated nucleation and crystal growth of perovskite films for solar cells with efficiency greater than $21 \%$. Nature Energy $1(10)$.

11. Brenner TM, et al. (2016) Conversion of single crystalline pbi2 to ch3nh3pbi3: Structural relations and transformation dynamics. Chemistry of Materials 28(18):6501-6510.

12. Guo Y, et al. (2015) Chemical Pathways Connecting Lead(II) lodide and Perovskite via Polymeric Plumbate(II) Fiber.

13. Patel JB, Milot RL, Wright AD, Herz LM, Johnston MB (2016) Formation Dynamics of CH3NH3Pbl3 Perovskite Following Two-Step Layer Deposition. Journal of Physical Chemistry Letters 7(1):96-102.

14. Barrit $\mathrm{D}$, et al. (2019) Impact of the solvation state of lead iodide on its two-step conversion to mapbi3: An in situ investigation. Advanced Functional Materials 29(47):1807544.

15. Sanches AW, Da Silva MA, Cordeiro NJ, Urbano A, Lourenço SA (2019) Effect of intermediate phases on the optical properties of Pbl2-rich $\mathrm{CH} 3 \mathrm{NH} 3 \mathrm{Pbl} 3$ organic-inorganic hybrid perovskite. Physical Chemistry Chemical Physics 21(9):5253-5261.

16. Liu J, et al. (2015) High-Quality Mixed-Organic-Cation Perovskites from a Phase-Pure Nonstoichiometric Intermediate (FAl)1-x-Pbl2 for Solar Cells. Advanced Materials 27(33):49184923

17. $\mathrm{Xu} \mathrm{X}$, et al. (2018) Air-processed mixed-cation Cs0.15FA0.85Pbl3 planar perovskite solar cells derived from a Pbl2-CsI-FAl intermediate complex. Journal of Materials Chemistry $A$ 6(17): $7731-7740$

18. Sanchez S, Steiner U, Hua X (2019) Phase Evolution during Perovskite Formation - Insight from Pair Distribution Function Analysis. Chemistry of Materials 31(9):3498-3506.

19. Pradeesh K, Baumberg JJ, Prakash GV (2009) In situ intercalation strategies for devicequality hybrid inorganic-organic self-assembled quantum wells. Applied Physics Letters 95(3):033309.

20. Jain SM, et al. (2016) Vapor phase conversion of Pbl2 to CH3NH3Pbl3: spectroscopic evidence for formation of an intermediate phase. Journal of Materials Chemistry A 4(7):26302642.

21. Laio A, Parrinello M (2002) Escaping free-energy minima. Proceedings of the National Academy of Sciences of the United States of America 99(20):12562-12566.

22. Barducci A, Bussi G, Parrinello M (2008) Well-tempered metadynamics: A smoothly converg ing and tunable free-energy method. Physical Review Letters 100(2).

23. Que CJ, et al. (2017) Perovskite-Like Organic-Inorganic Hybrid Lead lodide with a Large Organic Cation Incorporated within the Layers. Inorganic Chemistry 56(5):2467-2472.

24. Kamminga ME, de Wijs GA, Havenith RWA, Blake GR, Palstra TT (2017) The Role of Connectivity on Electronic Properties of Lead lodide Perovskite-Derived Compounds. Inorganic Chemistry 56(14):8408-8414.

25. Lermer C, et al. (2018) Completing the Picture of 2-(Aminomethylpyridinium) Lead Hybrid Perovskites: Insights into Structure, Conductivity Behavior, and Optical Properties. Chemistry of Materials 30(18):6289-6297.

26. Stoumpos CC, Mao L, Malliakas CD, Kanatzidis MG (2017) Structure-Band Gap Relationships in Hexagonal Polytypes and Low-Dimensional Structures of Hybrid Tin lodide Perovskites. Inorganic Chemistry 56(1):56-73.

27. Gratia P, et al. (2017) The Many Faces of Mixed Ion Perovskites: Unraveling and Understanding the Crystallization Process. ACS Energy Letters 2(12):2686-2693.

28. Ummadisingu A, Grätzel M (2018) Revealing the detailed path of sequential deposition for metal halide perovskite formation. Science Advances 4(2):e1701402.

29. Ummadisingu $\mathrm{A}$, et al. (2017) The effect of illumination on the formation of metal halide perovskite films. Nature 545(7653):208-212.

30. Harms HA, Tetreault N, Pellet N, Bensimon M, Gratzel M (2015) Mesoscopic photosystems for solar light harvesting and conversion: facile and reversible transformation of metal-halide perovskites. Faraday discussions 176:251-269.

31. Leguy AMA, et al. (2016) Dynamic disorder, phonon lifetimes, and the assignment of modes to the vibrational spectra of methylammonium lead halide perovskites. Phys. Chem. Chem. Phys. 18(39):27051-27066.

32. Daub M, Hillebrecht $H$ (2018) Tailoring the band gap in $3 d$ hybrid perovskites by substitution of the organic cations: (ch3nh3)1-2y(nh3(ch2)2nh3)2ypb1-yi3 $(0 \geq y \leq 0.25)$. Chemistry $-A$ European Journal 24(36):9075-9082.

33. Warren RF, Liang WY (1993) Raman spectroscopy of new lead iodide intercalation compounds. Journal of Physics: Condensed Matter 5(35):6407-6418.

34. Pellegrino G, et al. (2016) From Pbl2 to MAPbl3 through Layered Intermediates. The Journal of Physical Chemistry C 120(35):19768-19777.

35. Beckmann PA (2010) A review of polytypism in lead iodide. Crystal Research and Technology 45(5):455-460.

36. Khilji MY, Sherman WF, Wilkinson GR (1982) Raman study of three polytypes of pbi2. Journal of Raman Spectroscopy 13(2):127-133.

37. Zhang T, et al. (2019) Spontaneous low-temperature crystallization of $\alpha$-fapbi3 for highly efficient perovskite solar cells. Science Bulletin 64(21):1608-1616.

38. Dutta NS, Noel NK, Arnold CB (2020) The crystalline nature of colloids in methylammonium lead halide perovskite precursor inks revealed by cryo-electron microscopy. The Journal of Physical Chemistry Letters.

39. Snaith HJ, Eperon GE, Ball JM (2020) Two-step deposition process.

40. Coleman C, et al. (1996) Intercalation of hydrazines in lead iodide. Journal of Physics and Chemistry of Solids 57(6):1153-1158. Proceeding of the 8th International Symposium on Intercalation Compounds.

41. Caddeo C, Saba MI, Meloni S, Filippetti A, Mattoni A (2017) Collective Molecular Mechanisms in the CH3NH3 Pbl3 Dissolution by Liquid Water. ACS Nano 11(9):9183-9190.

42. Ryckaert JP, Ciccotti G, Berendsen HJ (1977) Numerical integration of the cartesian equations of motion of a system with constraints: molecular dynamics of n-alkanes. Journal of Computational Physics 23(3):327-341.

43. Plimpton S (1995) Fast Parallel Algorithms for Short-Range Molecular Dynamics. Journal of Computational Physics 117(1):1-19.

44. Bussi G, Donadio D, Parrinello M (2007) Canonical sampling through velocity rescaling. The 
Journal of Chemical Physics 126(1):014101.

45. Parrinello M, Rahman A (1981) Polymorphic transitions in single crystals: A new molecular dynamics method. Journal of Applied Physics 52(12):7182-7190.

46. Tribello GA, Bonomi M, Branduardi D, Camilloni C, Bussi G (2014) PLUMED 2: New feathers for an old bird. Computer Physics Communications 185(2):604-613.

47. Giannozzi P, et al. (2009) QUANTUM ESPRESSO: a modular and open-source software project for quantum simulations of materials. Journal of Physics: Condensed Matter 21(39):395502.

48. Perdew JP, et al. (2008) Restoring the Density-Gradient Expansion for Exchange in Solids and Surfaces. Physical Review Letters 100(13):136406.

49. Perdew JP, Burke K, Ernzerhof M (1996) Generalized gradient approximation made simple. Physical review letters 77(18):3865.

50. Grimme S (2006) Semiempirical gga-type density functional constructed with a long-range dispersion correction. Journal of computational chemistry 27(15):1787-1799.

51. Niu H, Piaggi PM, Invernizzi M, Parrinello M (2018) Molecular dynamics simulations of liquid silica crystallization. Proceedings of the National Academy of Sciences of the United States of America 115(21):5348-5352.

52. Debye P (1915) Zerstreuung von Röntgenstrahlen. Annalen der Physik 351(6):809-823.

53. Ahlawat $P$, et al. (2019) Atomistic mechanism of the nucleation of methylammonium lead iodide perovskite from solution. Chemistry of Materials 32(1):529-536.

54. Oganov AR, Ono S (2004) Theoretical and experimental evidence for a post-perovskite phase of MgSiO3 in Earth's D" layer. Nature 430(6998):445-448.

55. Li Y, et al. (2018) A Perovskite Electrolyte That Is Stable in Moist Air for Lithium-lon Batteries. Angewandte Chemie International Edition 57(28):8587-8591.

56. Ledinskyy M, et al. (2015) Raman spectroscopy of organic-inorganic halide perovskites. The journal of physical chemistry letters 6(3):401-406.

57. Sheng CX, et al. (2015) Laser action and photoexcitations dynamics in Pbl2 films. Optical Materials Express 5(3):530-537. 


\section{Supplementary Information for}

\section{Molecular dynamics simulations of two-step process enable room-temperature synthesis of $\alpha-\mathrm{FAPbl}_{3}$}

Paramvir Ahlawat, Haizhou Lu, Amita Ummadisingu, Haiyang Niu, Michele Invernizzi, Shaik Mohammed

Zakeeruddin, Anders Hagfeldt*, Michael Graetzel*, Ursula Rothlisberger*, and Michele Parrinello*

This PDF file includes:

Supplementary text

Figs. S1 to S6 


\section{Supporting Information Text}

a

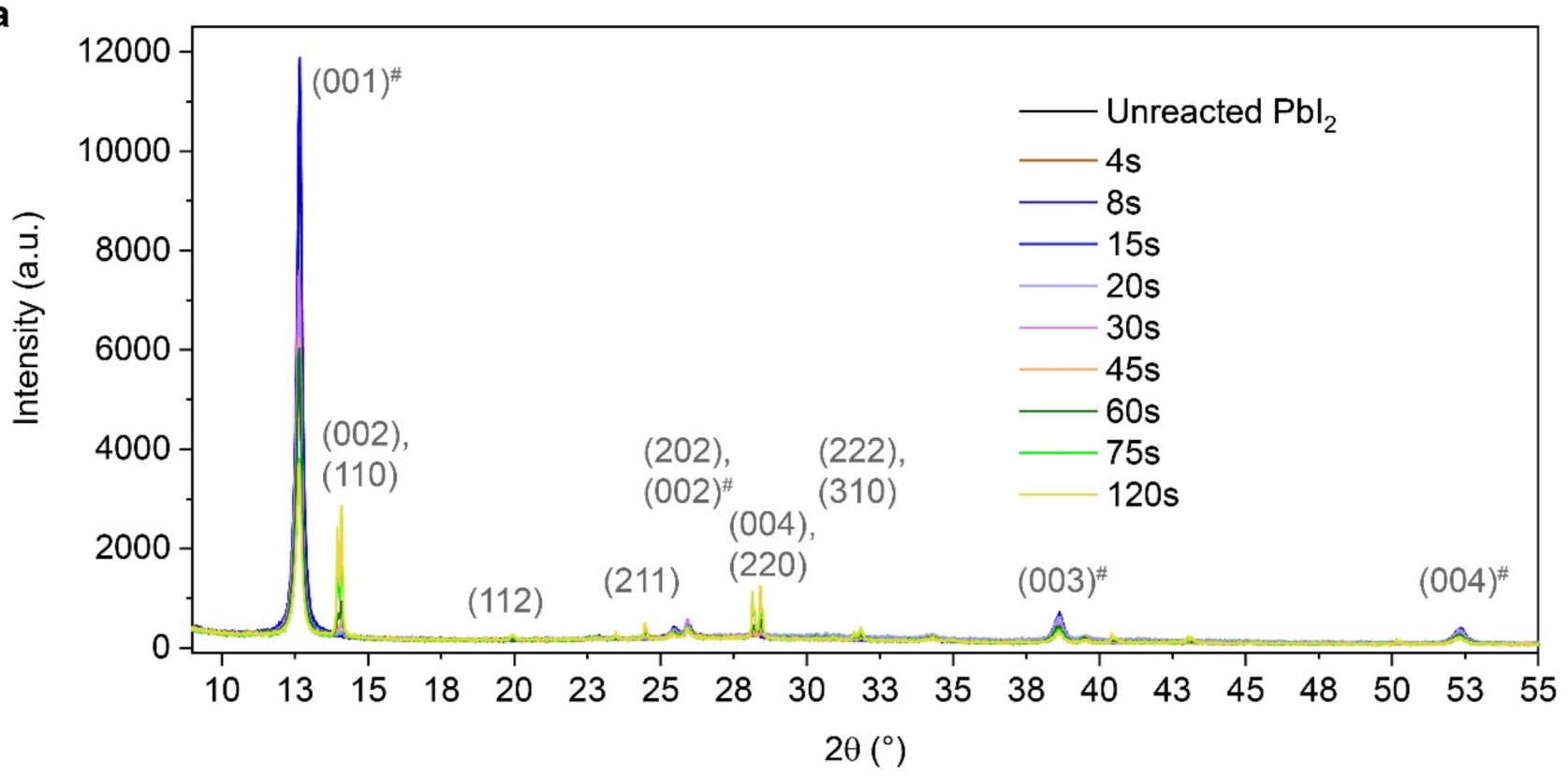

b

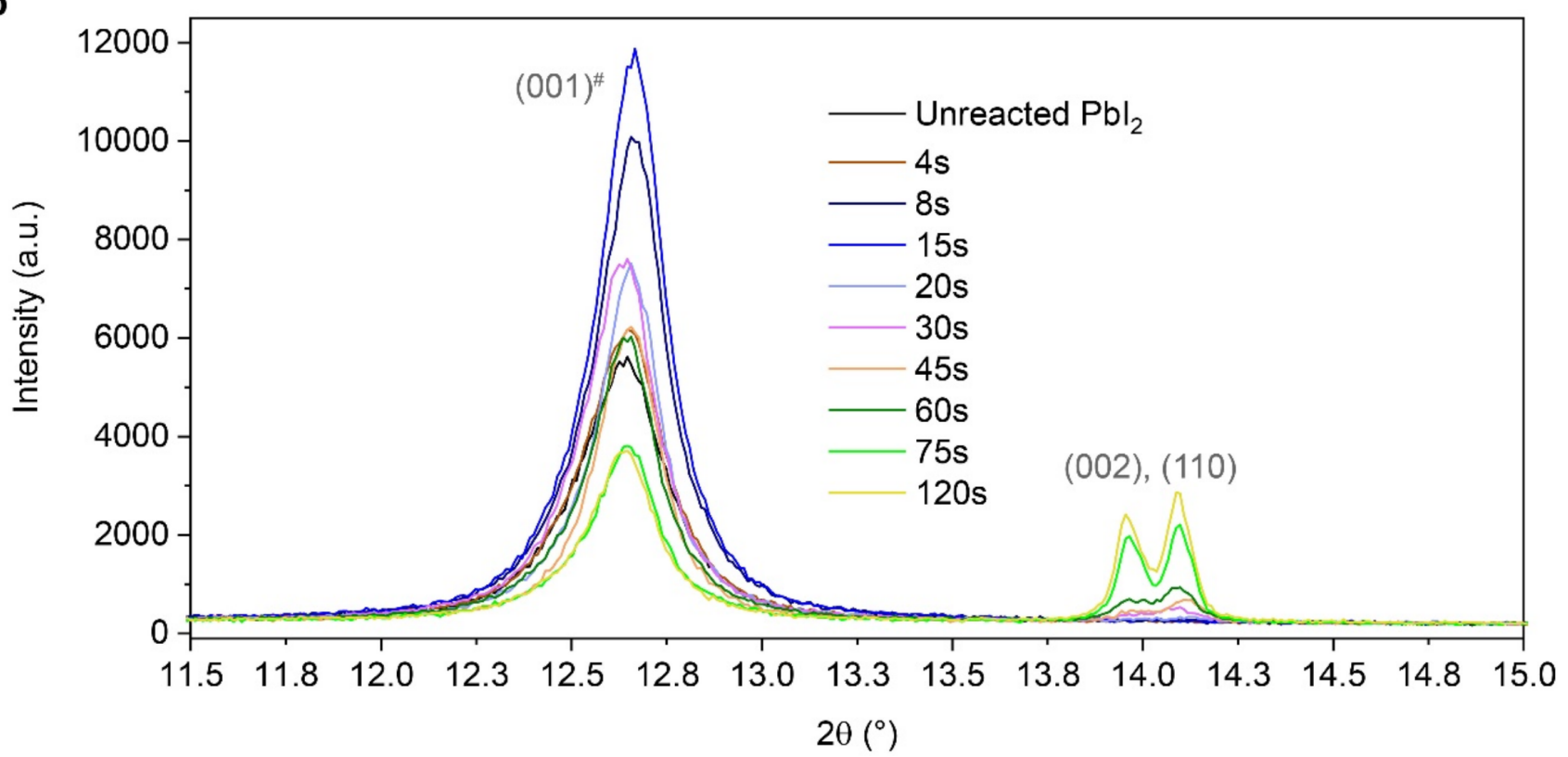

Fig. S1. XRD spectra monitoring the progress of $\mathbf{M A P b l}_{3}$ formation in the sequential deposition reaction Plots show an unreacted Pbl 2 sample and samples dipped in $5 \mathrm{mgml}^{-1}$ MAl solution for 4, 8, 15, 20, 30, 45, 60, 75 and 120s. Reflections of the $\mathrm{Pbl}_{2}$ in the $2 \mathrm{H}$ polytype are marked with \# while the remaining peaks are assigned based on the tetragonal phase of the $\mathrm{MAPbl}_{3}$. a) Full spectra showing all detected peaks. b) Zoom of a. 
12 layers

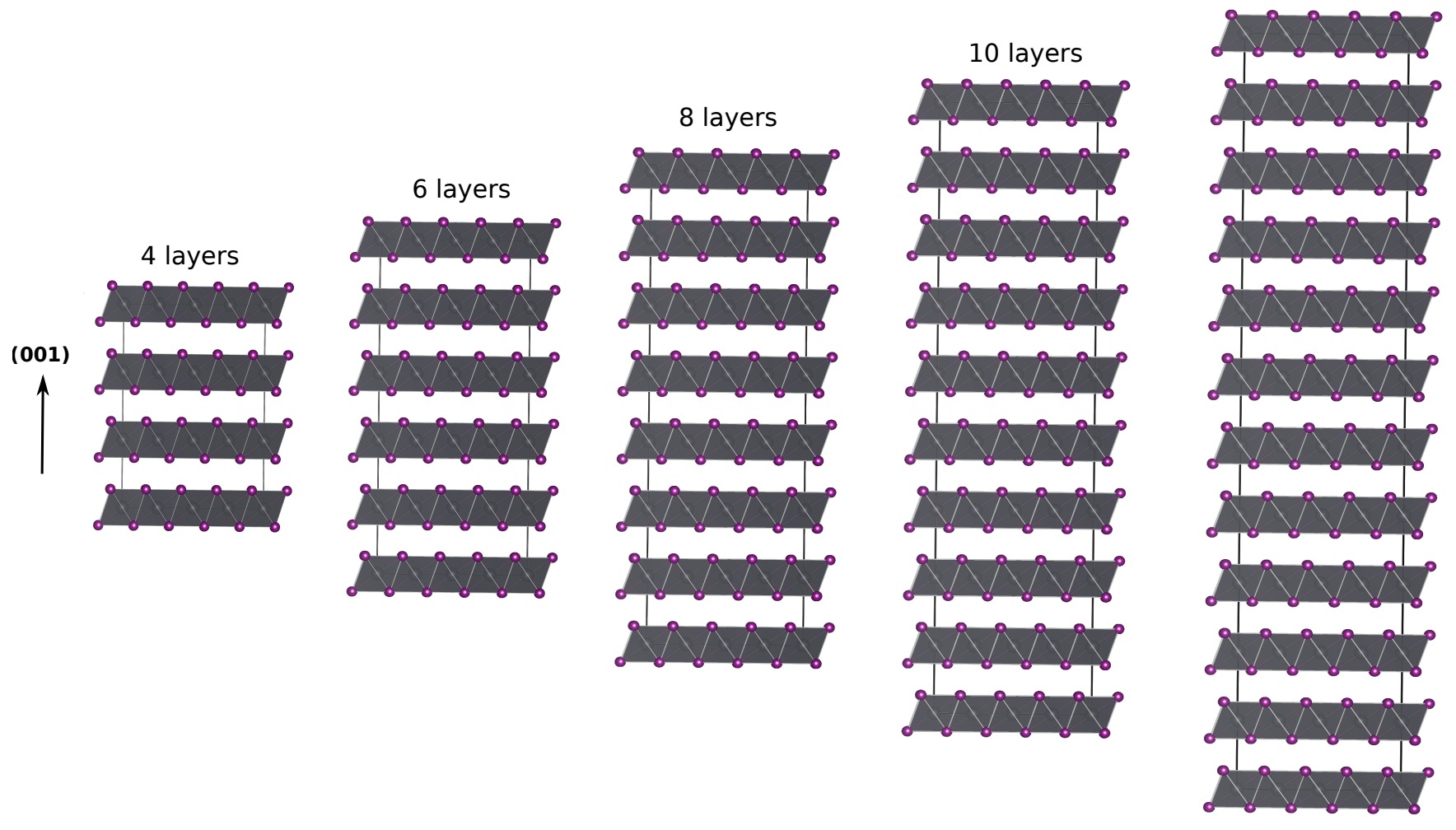

Fig. S2. $\mathrm{Pbl}_{2}$ supercells with different number of layers in (001) direction All supercells are represented with grey $\mathrm{Pb}-\mathrm{I}$ octahedra with $\mathrm{Pb}$ in center and I at corners with pink spheres. 

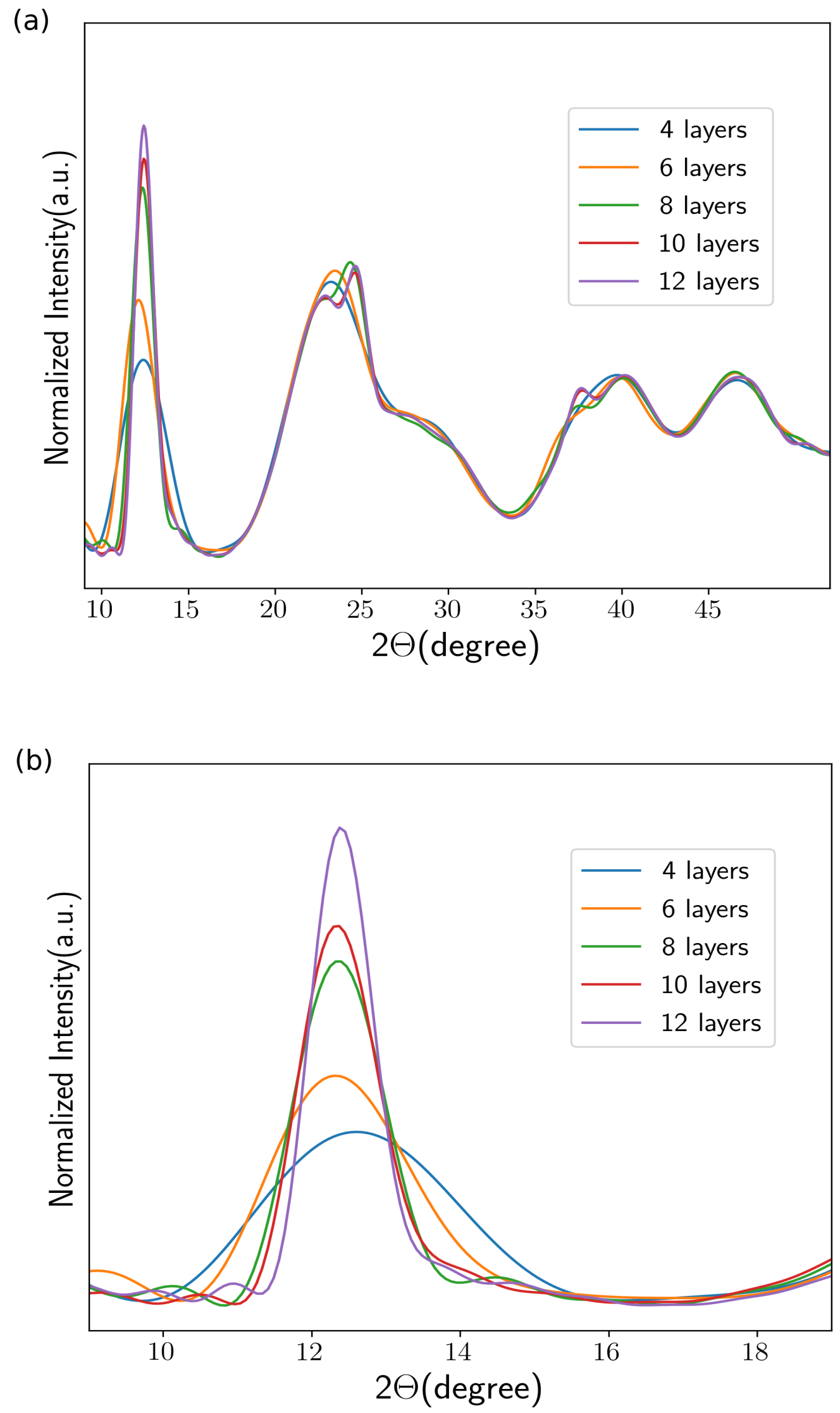


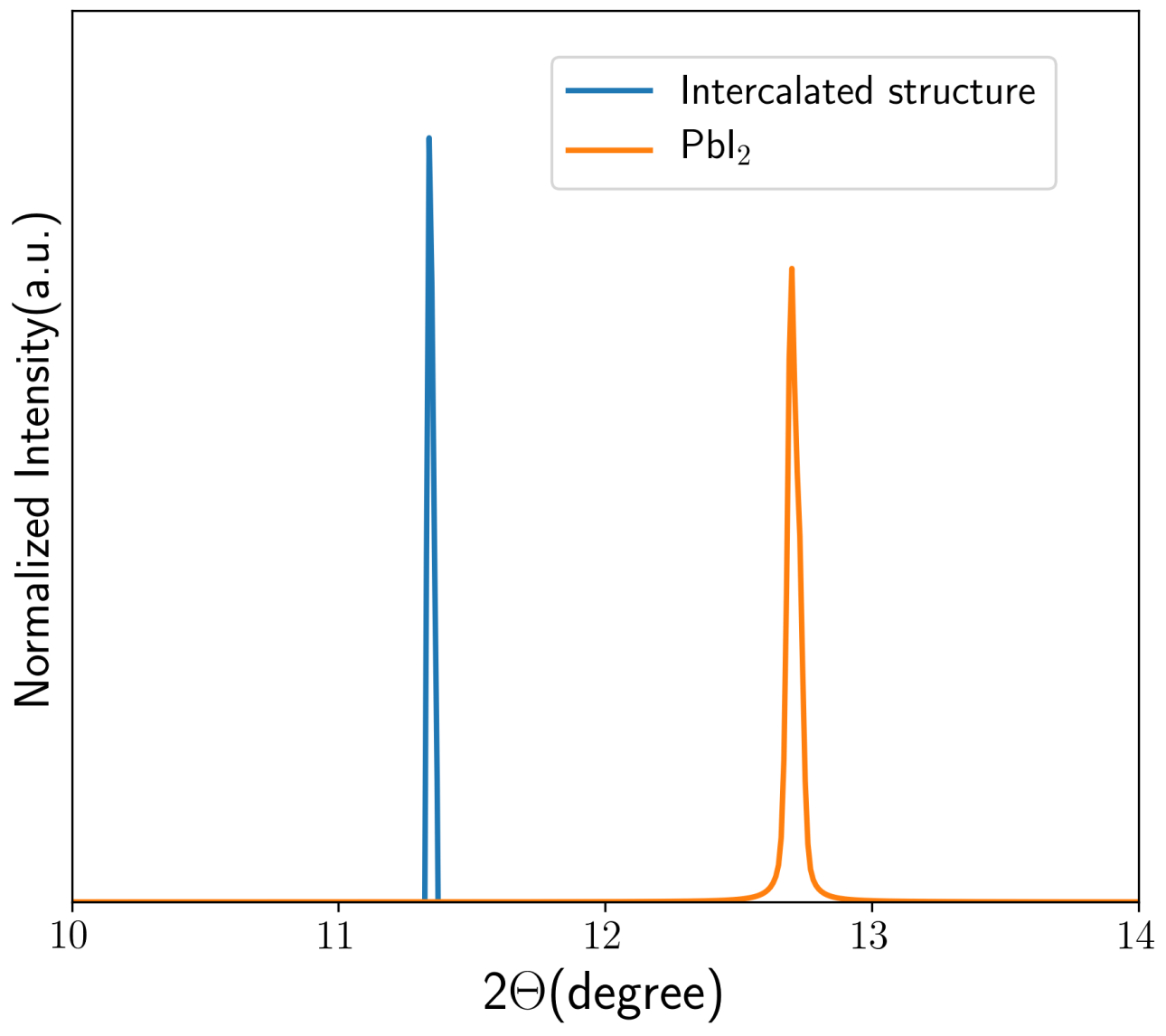

Fig. S4. Comparison of the first peak of powder diffraction patterns of DFT-D2 optimized $\mathrm{Pbl}_{2}$ and intercalated structure. We use VESTA software to calculate the powder diffraction patterns. 


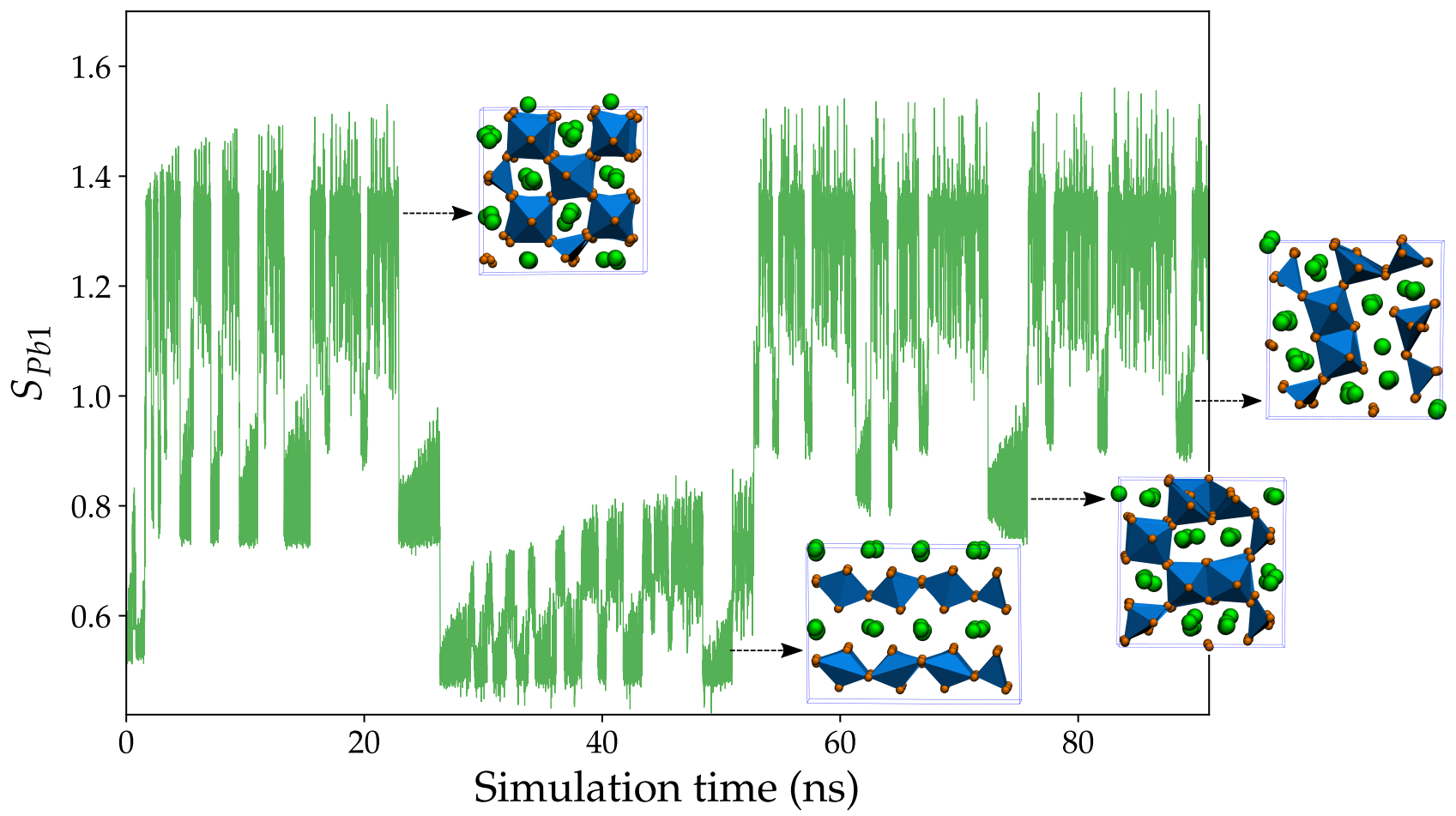

Fig. S5. Observed structures for $\mathrm{MAPbl}_{3}$ for smaller supercell. $\mathrm{Pb}-\mathrm{I}$ complexes are shown as golden polyhedra with $\mathrm{Pb}^{2+}$ in the center and $\mathrm{I}^{-}$on the corners. Free $\mathrm{I}^{-}$is shown as pink spheres. $\mathrm{MA}^{+}$ions are shown with ball and sticks with carbon as light blue, nitrogen as dark blue and hydrogens being white. 
a

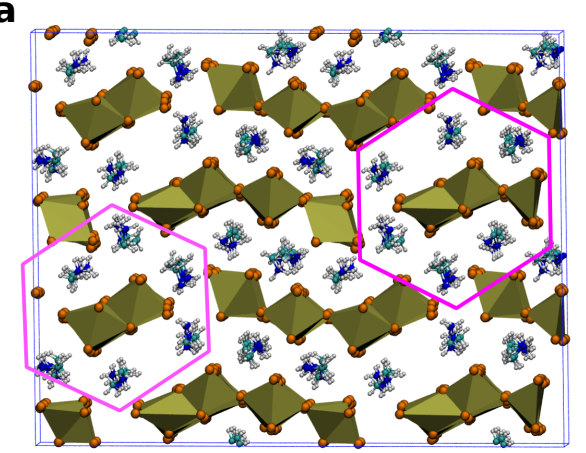

e

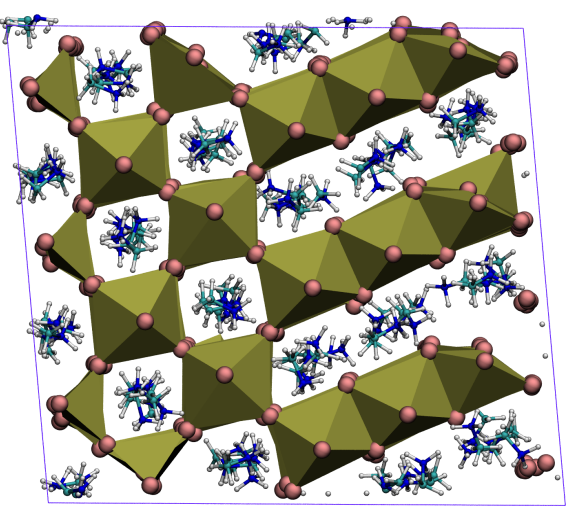

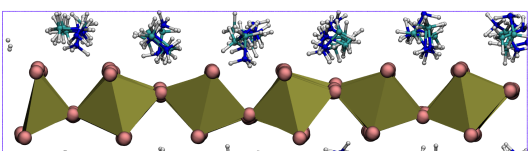

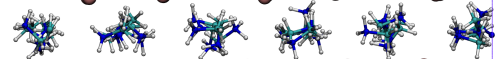

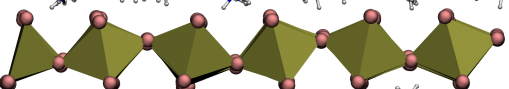

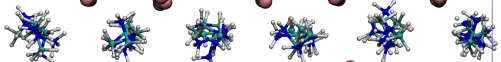

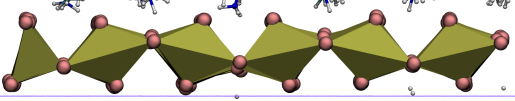

d

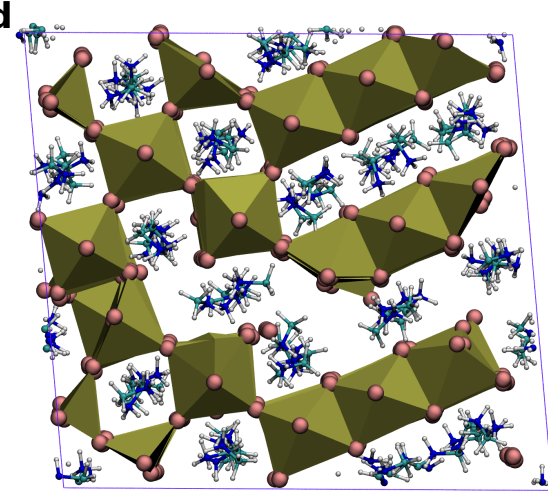

C

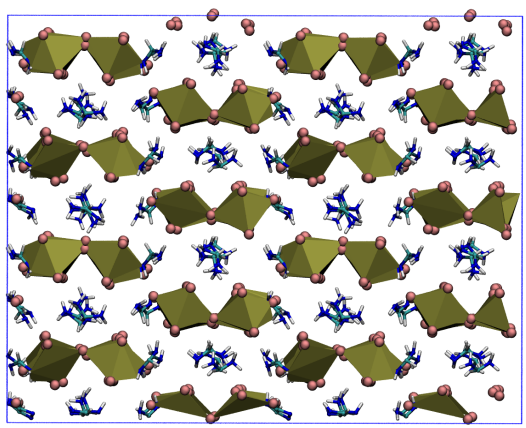

f

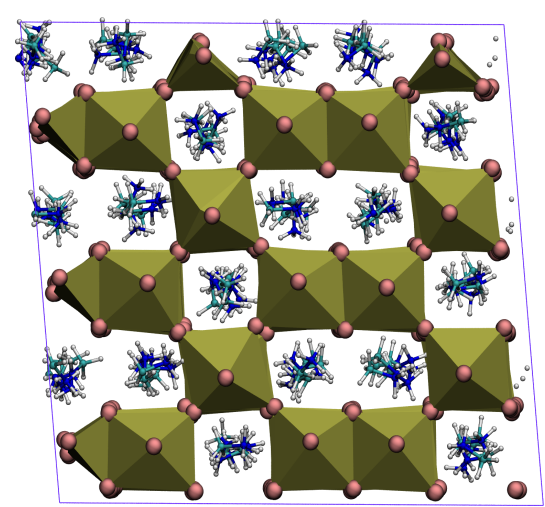

Fig. S6. Different polymorphs: $\mathrm{Pb}-\mathrm{I}$ complexes are shown as golden polyhedra with $\mathrm{Pb}^{2+}$ in the center and $\mathrm{I}^{-}$on the corners. Free $\mathrm{I}^{-}$is shown as pink spheres. $\mathrm{MA}^{+}$ions are shown with ball and sticks with carbon as light blue, nitrogen as dark blue and hydrogens being white. Hexagons are drawn in figure (a) to show the similarity to the solvent intercalated 1D phases during crysatllization of halide perovskites. 